\title{
Collagen Organization Critical Role in Wound Contraction
}

\author{
H. Paul Ehrlich ${ }^{1, *}$ and Thomas K. Hunt ${ }^{2}$ \\ ${ }^{1}$ Division of Plastic Surgery, Penn State University College of Medicine, Hershey, Pennsylvania. \\ ${ }^{2}$ Department of Surgery, University of California at San Francisco, San Francisco, California.
}

Background: Open wound closure by wound contraction produces a healed defect made up mostly of dermis. Generating thicker collagen fibers condenses granulation tissue, which pulls surrounding skin into the defect.

The Problem: What is the mechanism for open wound contraction? Is it through the generation of contractile force using sustained myosin ATPase, thus causing cell contraction or by rapid myosin ATPase that condenses collagen fibrils into fibers?

Basic/Clinical Science Addressed: The mechanism for wound contraction is not often debated after the discovery of the myofibroblast. Myofibroblasts are the major cell phenotype in maturing granulation tissue. It is concluded, not quite accurately, that myofibroblasts are responsible for wound contraction. As wound contraction progresses, polarized light microscopy reveals birefringence patterns associated with ever-increasing thickening of collagen fibers. Collagen fibers thicken by eliminating water between fibrils. Wound contraction requires collagen synthesis and granulation tissue compaction. Both myofibroblasts and fibroblasts synthesize collagen, but fibroblasts, not myofibroblasts, compact collagen. Free-floating fibroblast-populated collagen lattices (FPCL) contract by rapid myosin ATPase, thus resulting in thicker collagen fibers by elongated fibroblasts. The release of an attached FPCL, using sustained myosin ATPase, produces rapid lattice contraction, now populated with contracted myofibroblasts in the absence of thick collagen fibers. Discussion: In vivo and in vitro studies show that rapid myosin ATPase is the motor for wound contraction. Myofibroblasts maintain steady mechanotension through sustained myosin ATPase, which generates cell contraction forces that fail to produce thicker collagen fibers. The hypothesis is that cytoplasmic microfilaments pull collagen fibrils over the fibroblast's plasma membrane surface, bringing collagen fibrils in closer contact with one another. The self-assembly nature of collagen fixes collagen fibrils in regular arrays generating thicker collagen fibers.

Conclusion: Wound contraction progresses through fibroblasts generating thicker collagen fibers, using tractional forces; rather than by myofibroblasts utilizing cell contraction forces.

\section{BACKGROUND}

WOUND CONTRACTION, LITERALLY A shrinkage of open skin wounds, leaves a remarkably small scar as the surrounding normal skin moves centripetally to close the wound. It has aroused the curiosity of students of healing for many years (Fig. 1). For the most part, it is a benign and clinically beneficent process. It should not be confused with scar "contracture" that pulls deeper tissues, not only skin, toward the injury site, thus limiting normal motion; for

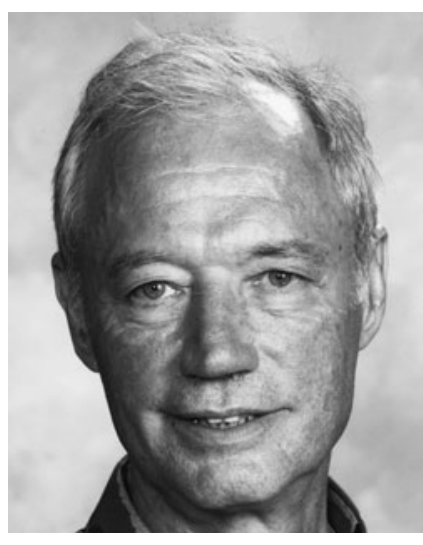

H. Paul Ehrlich

Submitted for publication August 8, 2011. *Correspondence: Division of Plastic Surgery, Penn State University College of Medicine, Hershey, Pennsylvania 17033 (e-mail: hehrlich@ psu.edu).

Abbreviations
and Acronyms
$\alpha \mathrm{SMA}=$ alpha smooth muscle
actin
$\mathrm{ADR}=$ attached delayed
released
$\mathrm{ECM}=$ extracellular matrix
$\mathrm{FF}=$ free floating
$\mathrm{FPCL}=$ fibroblast-populated
collagen lattice
$\mathrm{MLC}=$ myosin light chain
$\mathrm{MLCK}=$ myosin light chain
kinase
MLCP = myosin light chain
phosphatase


instance, deforming facial features or limiting the excursion of joints. The focus of this article is on the current concept of how this remarkable process occurs and how it precludes contracture.

With significant exceptions, wound contraction plays a limited role in human repair. However, if given enough time, even large open wounds of the human skull or posterior neck, for instance, can completely close by contraction alone usually leaving a small stellate area of neo-epithelization. These sites, and others in which contraction contributes to the final result, are subject to little movement or externally applied tension, and the surrounding skin should be loose and sufficiently flexible to yield the required length, and should be subject to little motion. Due to this property, most experimental data on wound contraction employ loose-skinned rodents.

\section{TARGET ARTICLES}

1. Berry DP, Harding KG, Stanton M, Jasani B, and Ehrlich HP: Human wound contraction: collagen organization, fibroblast and myofibroblasts. Plast Reconstr Surg 1998; 102: 124.

2. Levinson H, Moyer KE, Saggers GC, and Ehrlich HP: Calmodulin-myosin light chain kinase inhibition changes fibroblast-populated collagen lattice contraction, cell migration, focal adhesion formation, and wound contraction. Wound Repair Regen 2004; 12: 505.

3. Ehrlich HP and Rajaratnam JB: Cell locomotion forces versus cell contraction forces for collagen lattice contraction: an in vitro model of wound contraction. Tissue Cell 1990; 22: 407.

4. Dunphy JE and Udupa KN: Chemical and histochemical sequences in the normal healing of wounds. N Engl J Med 1955; 253: 847.

\section{CLINICAL PROBLEM ADDRESSED}

The focus is on understanding the process about how a full-thickness open wound closes by wound contraction, because it generally achieves a superior result when it can be enlisted. The hypothesis is that wound contraction results from cellular forces that organize collagen within granulation tissue in progressively shortened configurations until the open wound is closed.

\section{RELEVANT BASIC SCIENCE ADDRESSED}

The mechanism for wound contraction has been debated for many years. ${ }^{1}$ The current view first took
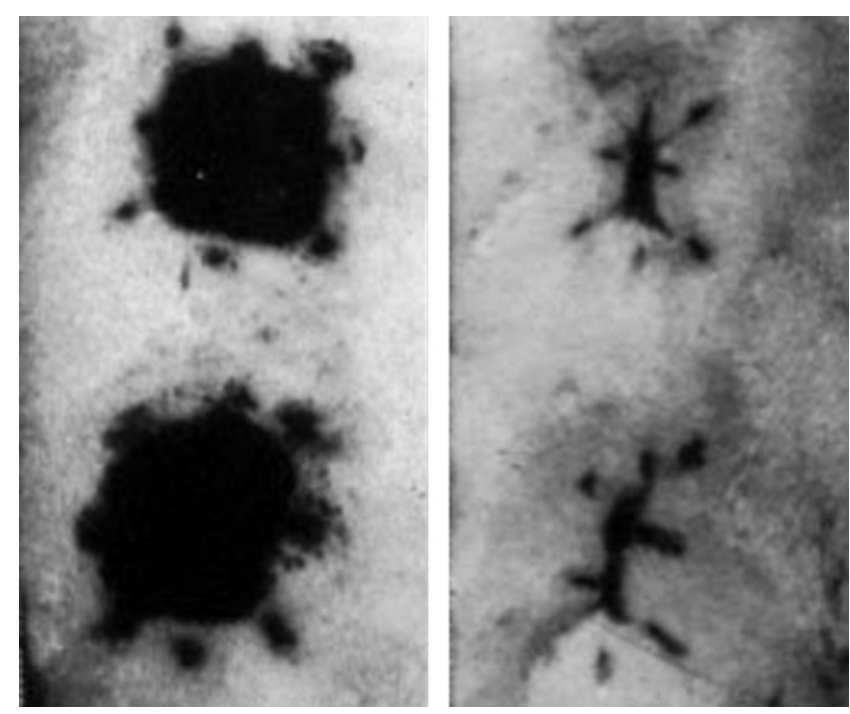

Figure 1. Contraction of a pair of tattooed full excision open wound in the dorsum of an adult rat. On the left is a pair of wounds at 1 day, and on the right is the same pair of wounds at day 18. Note that the tattoo marks at the corner of the wounds have moved very little, whereas the tattoo marks on the edges have moved to the center of the wound.

form when Gabbiani and coworkers ${ }^{2}$ discovered "myofibroblasts," which contain characteristics of both fibroblasts and smooth muscle cells in maturing granulation tissue, and concluded, not quite accurately, that contractility of these cells provides the force of wound contraction. The current preponderance of data indicates that cellular forces do participate, but they are provided mainly by fibroblast locomotion providing the forces that organizes newly deposited collagen in such a manner that collagen fibrils are fixed in a progressively more compact configuration by the elimination of water.

Myofibroblasts appear in normal as well as granulation tissue, a variety of fibrotic conditions, and are associated with tension. They are considered a cellular icon of fibrosis. A major identifying feature of the myofibroblast phenotype is alpha smooth muscle actin ( $\alpha$ SMA) within cytoskeletal stress fibers. However, full-thickness excisional wounds in rats contract by $50 \%$ in the first 7 days of healing before myofibroblasts first appear. A socalled proto-myofibroblast, containing cytoskeletal stress fibers, but not $\alpha$ SMA, by cell contraction forces has been proposed to explain this initial contraction. ${ }^{3}$ The identification of contracted myofibroblasts has been limited to released splinted wounds that have been physically restrained to contract. $^{4,5}$

Collagen deposition has been increasingly involved as a vital component. In vitro, soluble native collagen spontaneously polymerizes into a gel 
under physiologic conditions. In vivo, fibroblast locomotion forces direct the arrangement of collagen fibrils into thicker, longer fibers through their physical translocation. The orderly configuration of collagen fibrils in contraction results from their packing closer together by eliminating water. By this mechanism, small, loosely arranged, collagen fibrils in young granulation tissue are arranged by cell forces, and collagen self-assembly generates a denser matrix. This physical compaction of collagen is a major force in wound contraction. Contracted wounds are unique in their content of normal-appearing, large, birefringent fibers that are revealed by polarized light microscopy (Fig. 2).

Orderly packing of young collagen fibrils into growing collagen fibers requires rapid myosin ATPase activity. Myosin ATPase activity causes myosin to slide along an actin filament analogous to muscle contraction. Optimizing microfilament contraction requires phosphorylation of the myosin's regulatory peptide, myosin light chain (MLC). When MLC is phosphorylated at serine-19, myosin ATPase activity is optimized. ${ }^{6}$ This is under the control of myosin light chain kinase (MLCK), a key enzyme in rapid myosin ATPase activity, where ATP donates a phosphate to serine-19 (Fig. 3). With rapid myosin ATPase activity, there is a rapid turnover of the phosphorylated MLC through myosin light chain phosphatase (MLCP), which removes MLC serine-19 phosphate, a relaxation step required for myosin to again interact with MLCK. In contrast, the critical step for sustained myosin ATPase activity is the inhibition of MLCP that prevents the relaxation step and prolongs the longevity of
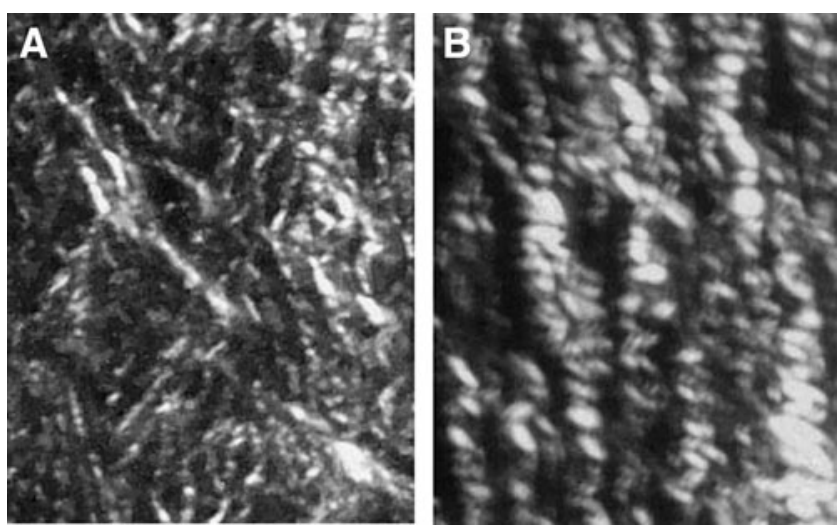

Figure 2. The birefringence pattern from Sirius red-stained polarized light microscopy of contracting open rat wounds is presented. (A) From young granulation tissue of a 5-day-old wound. (B) More mature granulation tissue from a 10-day-old contracting wound. Note a change in the diameters of collagen fibers. Magnification $40 \times$.

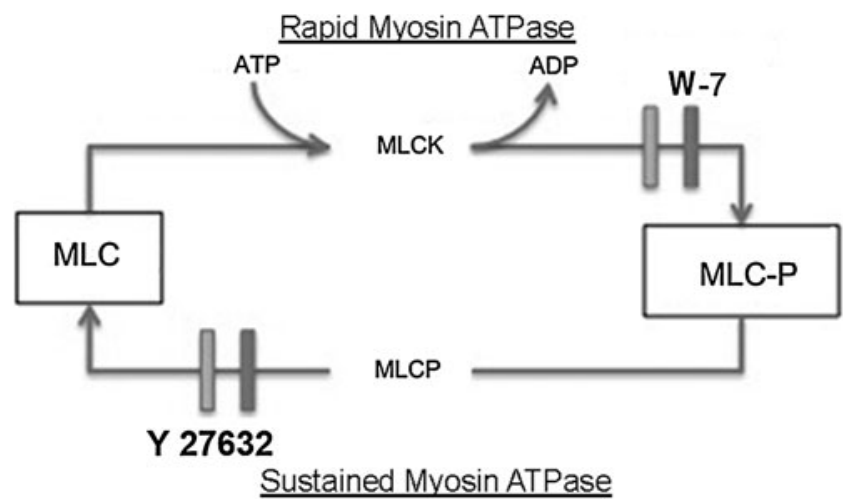

Figure 3. A diagram of the scheme for the rapid and sustained myosin ATPase is presented. The phosphorylation of MLC in rapid and sustained myosin ATPase activity occurs by different mechanisms. MLCK affixes a phosphate group from ATP onto MLC serine-19, which is necessary for optimal myosin ATPase activity. The inhibitor W-7 will block MLCK, thus inhibiting rapid myosin ATPase activity. MLCP removal of the phosphate group from MLC serine-19 is required for the relaxation step in cell locomotion through rapid myosin ATPase activity. Inhibiting MLCP activity, prolonging the phosphorylated state of MLC, defines the mechanism for sustained myosin ATPase activity, where MLCP is maintained in a chronic state. The inhibitor Y-27632 restores MLCP activity and terminates sustained myosin ATPase activity. MLC, myosin light chain; MLCK, myosin light chain kinase; MLCP, myosin light chain phosphatase.

MLC serine-19. ${ }^{7}$ This chronic, long-lived cytoskeletal microfilament contractile state is associated with contractile generated forces of smooth muscle cells within vascular and intestinal walls. Generated contractile forces from sustained myosin ATPase activity promote chronic tension and are independent of forces responsible for cell locomotion.

\section{EXPERIMENTAL MODELS}

\section{Animal studies}

The physical morphological attributes of myofibroblasts support their role as the critical cell for wound contraction. They contain robust cytoskeleton actin myosin stress fibers, as well as actin myosin filaments arranged along their plasma membranes, and commonly contain a wrinkled nucleus which suggests that they are in a contracted state. Mechanical tension within granulation tissue is demonstrated by making an incision along the wound edge, resulting in the immediate compaction of granulation tissue drawing it into the wound center, whereas surrounding skin falls away from the wound edge, demonstrating the opposing dynamic forces within a contracting wound. In vitro, the demonstration of sustained myosin ATPase activity utilized isolated strips of granulation tissue. When exposed to promoters of sustained myosin ATPase activity, they contract. $^{8}$ In contrast, in rat studies, wound 
contraction is inhibited by an MLCK inhibitor, which supports rapid myosin ATPase activity in wound contraction. ${ }^{9}$

Alizadeh and coworkers ${ }^{6}$ reported that retarded wound contraction was related to inhibited conversion of wound fibroblasts into myofibroblasts. However, other studies showed that wound contraction proceeds in the absence of myofibroblast populations. For instance, vanadate blocks protein tyrosine phosphatases and prevents the expression of $\alpha$ SMA within fibroblasts in full-thickness rat open wounds. ${ }^{10,11}$ Vanadate stops the inhibition of MLCP activity, which, in turn, blocks sustained myosin ATPase activity, inhibiting the appearance of myofibroblasts; while leaving the rate and degree of wound contraction unaffected. Thus, the lack of sustained myosin ATPase activity and the absence of myofibroblasts are not essential to wound contraction. ${ }^{12}$ It appears, therefore, that vanadate-treated wounds contract through rapid myosin ATPase activity. This supports the concept that the mechanism for wound contraction is through cell locomotion forces rather than through cell contraction forces. ${ }^{13}$

\section{Cell culture studies}

Rapid myosin ATPase activity in fibroblasts versus sustained myosin ATPase activity in myofibroblasts reveals differences in cell-cell and cell-extracellular matrix (ECM) interactions. Fibroblasts in monolayer culture plated at a low density on reaching confluence transform into myofibroblasts, and all cells become positive for $\alpha$ SMA within cytoskeletal stress fibers. ${ }^{14}$ Casting a collagen gel over a confluent monolayer of myofibroblasts eliminates cytoplasmic $\alpha$ SMA enriched stress fibers. ${ }^{12}$ This change in cell phenotype is related to a change in tension experienced by these cells with a reduction in tension associated with cells exclusively attached to a rigid plastic surface to an association with an elastic collagen matrix. Shared forces generated from cell-cell adherence become equivalent to the forces generated from the same cell adherence with its ECM. ${ }^{15}$ The strong tension generated between a myofibroblast with an underlying rigid surface is equivalent to the tension generated between a pair of adherent myofibroblasts. When a flexible collagen ECM associates with a myofibroblast on a rigid surface, the tension between the cell and its collagen gel ECM is reduced. The reduction of tension between cell and matrix will be also shared with an equivalent loss of intercellular tension between cell-cell associations. A loss of tension on myofibroblasts leads to their return to the fibroblast phenotype.
The fibroblast-populated collagen lattice (FPCL) contraction model introduced by Bell and cowork$\mathrm{ers}^{16}$ facilitated the study of wound contraction in cell culture. Bell's free-floating (FF)-FPCL undergoing contraction is exclusively populated with elongated fibroblasts with cytoskeletal microfilaments. For the most part, lattice compaction is completed in 2 days, exclusively populated with elongated fibroblasts. Investigating myofibroblasts in the FPCL contraction model were introduced by Tomasek and coworkers ${ }^{17}$ with the attached delayed released (ADR)-FPCL contraction model. Cast FPCLs that adhere to a tissue culture dish surface show no contraction until their physical release. At 4 days, ADR-FPCLs, exclusively populated with myofibroblasts, are released. Within 10 to $30 \mathrm{~min}$, contracted myofibroblasts populate the contracted lattice, which histologically is analogous to the contraction of a released splinted-open wound. A diagram of those differences in FF-FPCL contraction with ADR-FPCL contraction is presented in Fig. 4. The mechano-tension that develops in ADR-FPCL mimics the mechano-tension within granulation tissue. Vanadate and Y-27632 treated attached FPCLs show retarded ADRFPCL contraction. Vanadate averts the emergence of myofibroblasts by interfering with sustained myosin ATPase activity. Vanadate and Y-27632 interferes with neither FF-FPCL contraction nor rapid myosin ATPase activity. On the other hand, W-7 that inhibits MLCK blocking rapid myosin ATPase activity impedes FF-FPCL contraction. ${ }^{18}$ W-7 has no affect on ADR-FPCL contraction

FIBROBLAST POPULATED COLLAGEN LATTICE MODELS FF-FPCL $\underline{A D R-F P C L}$

\begin{tabular}{lll} 
Time lattice released: & $30 \mathrm{~min}$. & 4 Days \\
Time contraction starts: & $6 \mathrm{hrs}$ & $2 \mathrm{~min}$ \\
\hline Time contraction completed: & $48 \mathrm{hrs}$ & $1 \mathrm{hr}$ \\
Cell type and morphology: & $\begin{array}{r}\text { Fibroblasts } \\
\text { Elongated }\end{array}$ & $\begin{array}{r}\text { Myofibroblasts } \\
\text { Contracted }\end{array}$
\end{tabular}

Figure 4. A diagram identifying the differences in FF-FPCLs and ADRFPCLs is presented. The cell number, collagen concentration, and composition of culture medium are the same in both FPCLs. The difference is the time of releasing the lattice from the surface of the dish. FF, free floating; FPCL, fibroblast-populated collagen lattice; ADR, attached delayed released. 
via sustained myosin ATPase activity. Therefore, FF-FPCL contraction proceeds by rapid myosin ATPase activity and ADR-FPCL contraction through sustained myosin ATPase (Fig. 3). Thus, a relaxation phase is necessary for the continued generation of locomotive forces in fibroblasts.

Polarized light microscopy demonstrates differences in collagen organization between contracting FF-FPCL and ADR-FPCL. Collagen fibrils associated with elongated fibroblasts within contracting FF-FPCL that utilize rapid myosin ATPase activity appear as uniform, thicker, elongated fibers which are arranged parallel to the long axis of the cell (Fig. 5). Similar to open-wound contraction, contracting FF-FPCLs demonstrate the dynamic organization of collagen fibers with increasing intensity of birefringence. In contrast, collagen in contracted ADR-FPCLs fails to generate birefringence indicating absence of uniform packing of collagen fibers. As lattice contraction proceeds, contracting myofibroblasts create the accumulation of disorganized collagen fibers, which is in contrast to the uniform organized collagen fibers found within granulation tissue from contracting wounds. To summarize, in the actively contracting FF-FPCL, neither myofibroblasts with $\alpha$ SMA stress fibers nor contracted cells are present, and collagen is arranged into thicker,

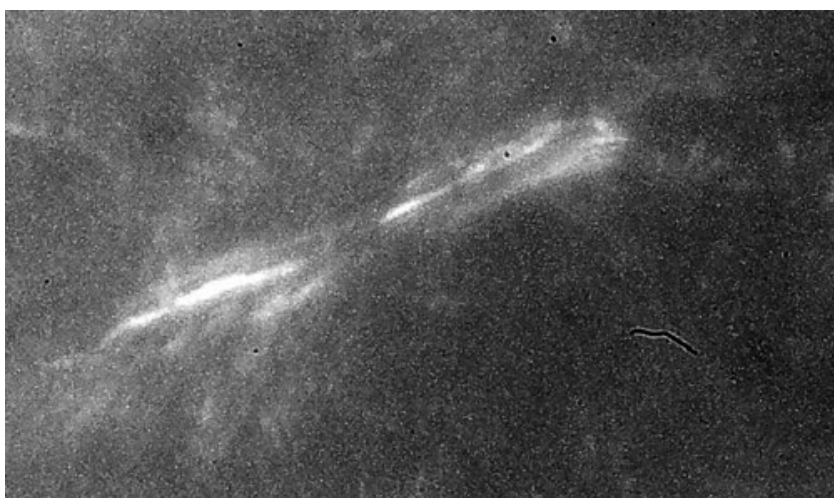

Figure 5. The birefringence pattern from polarized light microscopy from a 1-day-old contracting FF-FPCL that shows the birefringence pattern from parallel aligned collagen fibers localized at the ends of an elongated fibroblast. With polarized light microscopy, minimal cellular morphology is achieved. Fibroblasts translocate collagen fibrils by passing them over their plasma membrane surface, aligning them in parallel arrays. Magnification $80 \times$. longer collagen fibers demonstrated by polarized light microscopy birefringence.

\section{DISCUSSION}

the fact that cell locomotion through tractional forces via rapid myosin ATPase activity is the mechanism for wound contraction is supported by in vivo and in vitro experiments. The generation of cell locomotion forces requires dynamic microfilaments that contract and relax. The process of maintaining steady mechano-tension forces requires maintaining microfilaments within stress fibers under constant tension that avoid relaxation. Wound contraction requires the uniform packing of collagen fibrils into collagen fibers through rapid myosin ATPase activity. Collagen fibrils linked to microfilaments are dragged over the fibroblast's plasma membrane surface without distorting the cell's shape and through collagen fibrils incorporation into growing collagen fibers, they become a part of the granulation tissue connective tissue matrix. This proposed mechanism is similar to a ratchet tightening a bolt. When a ratchet is rotated in a clockwise direction, it tightens the bolt but 
when rotated in the opposite direction, there is no movement of the bolt, because the bolt remains fixed, and the ratchet is disengaged from applying force to the bolt. In contrast, sustained myosin ATPase activity is similar to a wrench tightening a bolt; rotating the wrench in a clockwise direction tightens the bolt, but rotating that wrench in a counterclockwise direction, while still engaged with the bolt, will loosen it. When fibroblast microfilaments relax, the collagen fibrils do not return to the random arranged collagen fibrils pool, but are locked into a stable collagen fiber. The microfilaments can engage in the translocation of new collagen fibrils and their incorporation into a growing collagen fiber. As tension builds between granulation tissue and surrounding dermis, which takes about 7 days, wound fibroblasts transform into myofibroblasts, which stabilizes the progress made by the organization of granulation tissue ECM, through fibroblasts giving rise to uniform organized collagen fibers.

\section{INNOVATION}

The closure of a full-thickness wound by wound contraction is a slow process and not commonly used. Skin grafting is fast and effective in closing a defect. However, grafting, a surgical procedure, creates a new wound that causes discomfort and poses cosmetic issues. New therapies that lead to faster wound contraction should reduce the need for grafting. The development of more efficient wound contraction, possibly by enhancing the rate of collagen organization that would hasten the rate of contraction and eliminate both a surgical pro- cedure and a secondary defect and scar, would be welcome.

\section{SUMMARY ILLUSTRATION}

Comparing myofibroblasts and fibroblasts in wound contraction

\begin{tabular}{ll}
\hline \multicolumn{1}{c}{ Myofibroblasts } & \multicolumn{1}{c}{ Fibroblasts } \\
\hline Cytoskeletal stress fibers with $\alpha$ SMA & Cytoskeletal microfilaments \\
Stationary cells & Locomotive cells \\
No collagen organization & Organizes collagen \\
Sustained myosin ATPase & Rapid myosin ATPase \\
\hline
\end{tabular}

\section{CAUTION, CRITICAL REMARKS, AND RECOMMENDATIONS}

Most of the work presented is drawn from cell culture and in animal studies. No one has to be reminded of the successes of growth factors in the laboratory in promoting wound healing in animal models and their limited success in the clinic. Reliance on contraction is not practical for large defects and is time consuming. Making the best selection requires experienced clinical judgment.

\section{ACKNOWLEDGMENTS AND FUNDING SOURCES}

No funding sources to acknowledge.

\section{AUTHOR DISCLOSURE AND GHOSTWRITING}

No disclosures. No ghostwriters were used to write this chapter.

\section{REFERENCES}

1. Dunphy JE and Udupa KN: Chemical and histochemical sequences in the normal healing of wounds. N Engl J Med 1955; 253: 847.

2. Gabbiani G, Ryan GB, and Majne G: Presence of modified fibroblasts in granulation tissue and their possible role in wound contraction. Experientia 1971; 27: 549.

3. Tomasek JJ, Gabbiani G, Hinz B, Chaponnier C, and Brown RA: Myofibroblasts and mechanoregulation of connective tissue remodelling. Nat Rev Mol Cell Biol 2002; 3: 349 .

4. Hinz B and Gabbiani G: Mechanisms of force generation and transmission by myofibroblasts. Curr Opin Biotechnol 2003; 14: 538.

5. Gabbiani G: The myofibroblast in wound healing and fibrocontractive diseases. J Pathol 2003; 200: 500 .
6. Adelstein RS: Calmodulin and the regulation of the actin-myosin interaction in smooth muscle and nonmuscle cells. Cell 1982; 30: 349.

7. Somlyo AP and Somlyo AV: Signal transduction by G-proteins, rho-kinase and protein phosphatase to smooth muscle and non-muscle myosin II. J Physiol 2000; 522: 177.

8. Gabbiani G, Hirschel BJ, Ryan GB, Statkov PR, and Majno G: Granulation tissue as a contractile organ. A study of structure and function. J Exp Med 1972; 135: 719

9. Levinson H, Moyer KE, Saggers GC, and Ehrlich HP: Calmodulin-myosin light chain kinase inhibition changes fibroblast-populated collagen lattice contraction, cell migration, focal adhesion formation, and wound contraction. Wound Repair Regen 2004; 12: 505.
10. Ehrlich HP, Keefer KA, Myers RL, and Passanit A: Vanadate and the absence of myofibroblasts in wound contraction. Arch Surg 1999; 134: 494

11. Mackay DJ, Moyer KE, Saggers GC, Myers RL, Mackay DR, and Ehrlich HP: Topical vanadate optimizes collagen organization within granulation tissue. Wound Repair Regen 2003; 11: 204.

12. Ehrlich HP, Allison GM, and Leggett M: The myofibroblast, cadherin, alpha smooth muscle actin and the collagen effect. Cell Biochem Funct 2006; 24: 63

13. Ehrlich HP and Rajaratnam JB: Cell locomotion forces versus cell contraction forces for collagen lattice contraction: an in vitro model of wound contraction. Tissue Cell 1990; 22: 407. 
14. Masur SK, Dewal HS, Dinh TT, Erenburg I, and Petridou S: Myofibroblasts differentiate from fibroblasts when plated at low density. Proc Natl Acad Sci U S A 1996; 93: 4219.

15. Maruthamuthu V, Sabass B, Schwarz US, and Gardel ML: Cell-ECM traction force modulates endogenous tension at cell-cell contacts. Proc Natl Acad Sci U S A 2011; 108: 4708.
16. Bell $E$, Ivarsson $B$, and Merrill C: Production of a tissue-like structure by contraction of collagen lattices by human fibroblasts of different proliferative potential in vitro. Proc Natl Acad Sci U S A 1979; 76: 1274.

17. Tomasek JJ, Haaksma CJ, Eddy RJ, and Vaughan MB: Fibroblast contraction occurs on release of tension in attached collagen lattices: dependency on an organized actin cytoskeleton and serum. Anat Rec 1992; 232: 359.

18. Ehrlich HP, Sun B, Kainth KS, and Kromath F: Elucidating the mechanism of wound contraction: Rapid versus sustained myosin ATPase activity in attached-delayed-released compared with freefloating fibroblast-populated collagen lattices Wound Repair Regen 2006; 14: 625. 